\title{
Si/SiGe near-infrared photodetectors grown using low pressure chemical vapour deposition
}

\author{
P. Iamraksa $\cdot$ N. S. Lloyd $\cdot$ D. M. Bagnall
}

Published online: 5 June 2007

(C) Springer Science+Business Media, LLC 2007

\begin{abstract}
Near-infrared photodetectors have been fabricated using standard CMOS processes in conjunction with the multilayer growth of $\mathrm{Si} / \mathrm{SiGe}_{0.06}$ using low-pressure chemical vapor deposition (LPCVD). Cross-section scanning electron microscopy (SEM) indicates the existence of quantum dot like corrugations in devices with particularly thick $\mathrm{SiGe}_{0.06}$ quantum wells. With an accumulation of germanium atoms at the crest of such features and commensurate high germanium concentration we see a considerable enhancement of the long wavelength detection sensitivity of photodetectors in the range 1100-1300 nm. By fitting experimental data the minimum energy gap of the structure is found to be $0.88 \mathrm{eV}$ corresponding to a germanium concentration of around $15 \%$.
\end{abstract}

\section{Introduction}

Silicon germanium has strong potential for use in siliconbased optoelectronics devices, particularly in the field of infrared photodetectors. The most challenging obstacles to widespread utilization of $\mathrm{SiGe}$ photodetectors are the epitaxial difficulties that arise as a result of the lattice mismatch between silicon and silicon germanium with a

P. Iamraksa $(\bowtie) \cdot$ D. M. Bagnall

Nanoscale Systems Integration, Electronics and Computer Science, University of Southampton, Southampton SO17 1BJ, UK

e-mail: pi01r@ecs.soton.ac.uk

N. S. Lloyd

Innos Ltd, Millbrook Technology Campus,

Southampton SO15 ODJ, UK high germainium content. Lattice constant difference between silicon and germanium is nearly $4 \%$, and thus significantly higher than other material systems commonly used within band-engineered devices such as. GaAs and AlGaAs. Nevertheless, the growth of high-quality epitaxial SiGe has progressed considerably over the last 20 years, particularly as $\mathrm{Si} / \mathrm{SiGe}$ heterojunction bipolar transistor and high electron mobility transistors have encouraged commercial use of SiGe epitaxy systems.

The fundamental structural, optical and electronic characteristics of the SiGe system are well known [1-3]. With suitable thickness, well below critical thickness, a $\mathrm{SiGe}$ quantum well can provide a smaller energy gap than SiGe alloy obtained by either bulk growth or thick epitaxial growth $[4,5]$. These band-gap modifications are a result of both the presence of strain induced in the SiGe and the presence of quantum confinement ability. The ability to modify the band-structure by alloying, strain effects and quantum confinement promises great potential for $\mathrm{Si}$ optoelectronics where devices can be designed to operate in the mid to far infra-red $[6,7]$.

Strain build-up prevents the optimum usage of high-Ge content SiGe layers beyond certain critical thicknesses. In fact thicknesses of only a few nanometers thick can be grown, otherwise dislocation networks are introduced into layers, and the electrical properties of devices are considerably degraded. To overcome critical thickness limits, multi-layers of $\mathrm{Si}$ and $\mathrm{SiGe}$ are used to ensure a sufficient volume of low-bandgap material. In this work we have used low pressure chemical vapour deposition to produce epitaxial $\mathrm{Si} / \mathrm{SiGe}$ multilayers in order to maximize long wavelength detection for silicon based devices.

Several epitaxial growth techniques can be used to realize strained $\mathrm{SiGe}$ heterostructures. The most popular methods are molecular beam epitaxy (MBE) and chemical 
vapor deposition (CVD). CVD methods provide high growth rate, and they are compatible with industrial silicon device manufacturing processes. There are various CVD designs depending on the different epitaxy requirements. For the growth of $\mathrm{SiGe}$ on $\mathrm{Si}$ substrates, ultra-high vacuum CVD [8-10], rapid thermal CVD, remote plasma-enhanced CVD [11], and other low pressure CVD techniques have been reported [12]. The apparatus used in this report, is low pressure CVD equipment, designed and built at Southampton University [15].

SiGe epitaxial growth can progress in two types of regime: two-dimensional (2D) $\mathrm{SiGe}$ layer growth and three-dimensional (3D) "island" growth. Layered, 2D SiGe provides near-ir sensitivity in the range $1.3-1.5 \mu \mathrm{m}$, depending on Ge content, mid- and far-ir sensitivity can also be realized by 2D SiGe [13]. Meanwhile, 3D SiGe islands provides the possibility of enhanced sensitivity in the near- and mid- IR ranges. To produce 3D SiGe nanostructures, surface controlled reactions and nucleations must be considered and self-assembled growth, exploiting Ge surface segregation and strain relaxation under specific growth conditions and relatively low temperature $[12,14]$.

\section{Experiment procedure}

A series of $\mathrm{p}$-i-n $\mathrm{Si} / \mathrm{SiGe}$ photodetectors were fabricated on p-type $<100>$ substrates $\left(17-33 \mathrm{ohm} / \mathrm{cm}^{2}\right)$ by predominantly standard CMOS processes. Devices were designed with a deep n-well guard-ring, produced by phosphorous implantation. Shallow $\mathrm{p}^{+}$doping was achieved by boron implantation into the substrate. The series of low-pressure chemical vapor deposition (LPCVD) epitaxial growths were carried out at $820^{\circ} \mathrm{C}$ and 0.4 Torr growth pressure [15]. The epitaxial layers were deposited without doping, and consisted of a $100 \mathrm{~nm}$ silicon buffer, 10 periods of $\mathrm{Si} / \mathrm{SiGe}_{0.06}$, and then a $100 \mathrm{~nm}$ silicon cap layer. The variations of $\mathrm{Si} / \mathrm{SiGe}_{0.06}$ multilayer are defined in Table 1 . A shallow $n^{+}$layer was then implanted into cap layer. Plasma etching was used to form a device mesa and a passivating oxide, made of Borophosilicate glass (BPSG), was deposited by plasma enhanced chemical vapor deposition (PECVD). Device contacts were formed by sputter

Table 1 Nominal composition, nominal SiGe thickness, band gap energy and phonon energy of investigated devices

\begin{tabular}{lllll}
\hline Structure & $\begin{array}{l}\text { Nom. } \\
\text { composition }\end{array}$ & $\begin{array}{l}\text { Nom. SiGe } \\
\text { thickness }\end{array}$ & $E_{g}(\mathrm{eV})$ & $E_{p h}(\mathrm{meV})$ \\
\hline $2675-1$ & 0.06 & 10 & 0.885 & 45 \\
$2675-2$ & 0.06 & 20 & 0.885 & 45 \\
$2675-3$ & 0.06 & 30 & 0.865 & 65 \\
\hline
\end{tabular}

deposited titanium/aluminum (Ti/Al) that were then patterned at the top and the bottom of the device mesa by optical lithography. The device configuration and perspective are shown in Fig. 1a and b. Device cross-section and device perspective were performed by thermal field emission scanning electron microscope (FEGSEM) (Jeol JSM-6500F), and photoconductivity was performed by standard white-light (tungsten) excitation with a lock-in technique.

\section{Results and discussion}

The FEGSEM provides image resolution of $1.5 \mathrm{~nm}$ and, as a consequence can provide useful information without the need for relatively time consuming transmission electron microscopy (TEM) techniques. Cross-sections of our three device layers are shown in Fig. 2.

In all images the 10 periods of $\mathrm{Si} / \mathrm{SiGe}_{0.06}$ are well resolved. The bright white bands represent the $\mathrm{Si}_{1-x} \mathrm{Ge}_{x}$ layers, and the darker bands represent silicon. We can see the development of corrugations in all three devices layers, though this is least obvious in 2675-1 Fig. 2a. The FEGSEM images indicate that the level of corrugation increases with increasing $\mathrm{Si}_{1-x} \mathrm{Ge}_{x}$ thickness. Meanwhile, the surface appearance of the devices, as observed by Normarski microscope are also indicative or 3D growth resulting from increased $\mathrm{Ge}$ content. The corrugated features of the thicker $\mathrm{Si}_{1-x} \mathrm{Ge}_{x}$ layers in Fig. 2c seem to reduce the local thickness of the commensurate silicon spacer layers, in a way that is reminiscent of commonly observed $\mathrm{Si}_{1-x} \mathrm{Ge}_{x}$ self-assembled quantum-dot and wetting layer structures [16]. The discontinuous feature in Fig. 2b probably originated during sample cleaving, dislocation networks are not observed in 2675-2.

The lattice mismatch between silicon and germanium yields elastic strain during the growth process. High-temperature and high adsorption rates lead to a increasing probability of $\mathrm{Ge}$ adatoms nucleating with other Ge adatoms, this is because adatom (diffusion) lifetimes are relatively long and surface concentrations will be relatively high. Under these conditions layer-by-layer epitaxial growth is less likely than $3 \mathrm{D}$ growth modes.

Figure 3 shows normalized photocurrent (PC) versus wavelength for our devices compared with a similar silicon device. We can see that all devices exhibit good diode characteristics (Fig. 3, inset). The sensitivity of all three SiGe devices can be seen to extend further into the IR than the silicon device as a result of the $\mathrm{Si}_{1-x} \mathrm{Ge}_{x}$ quantum wells, the cut-off for each device occurs at around $1300 \mathrm{~nm}$ $(0.955 \mathrm{eV})$. Enhancement of PC into the IR particularly in the range $1100-1300 \mathrm{~nm}$ is expected as a result of alloying as well as the presence of the strain and quantum 
Fig. 1 (a) Schematic diagram of $\mathrm{Si} / \mathrm{SiGe}$ photodetectors and (b) SEM of device mesas and cointacts
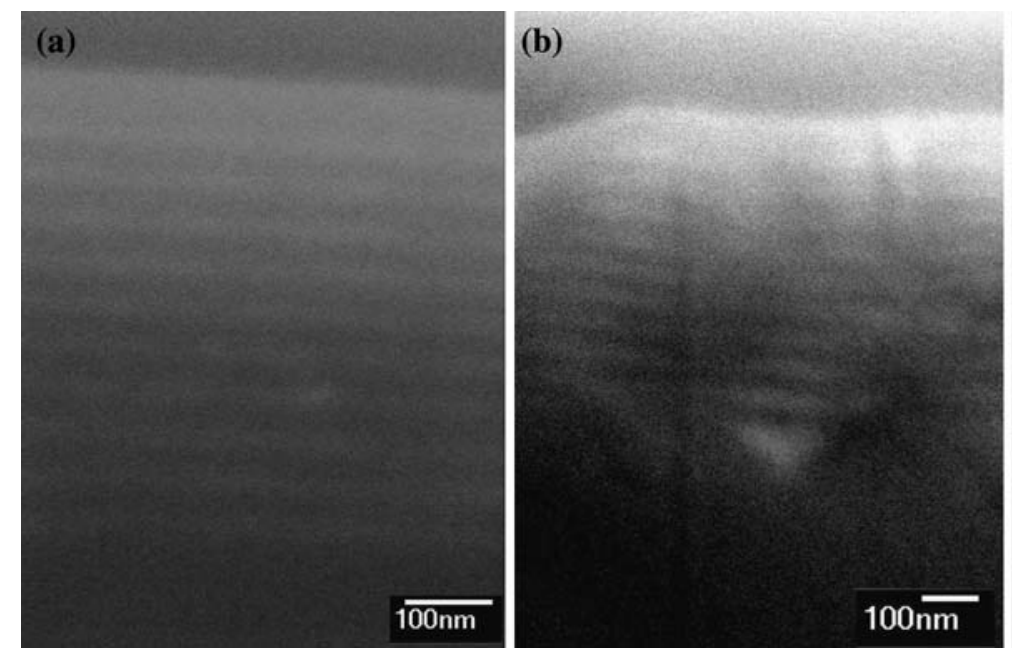

Fig. 2 Cross section SEM images of investigated devices. (a), (b) and (c) are representative sections of devices 2675-1, -2 and -3 respectively

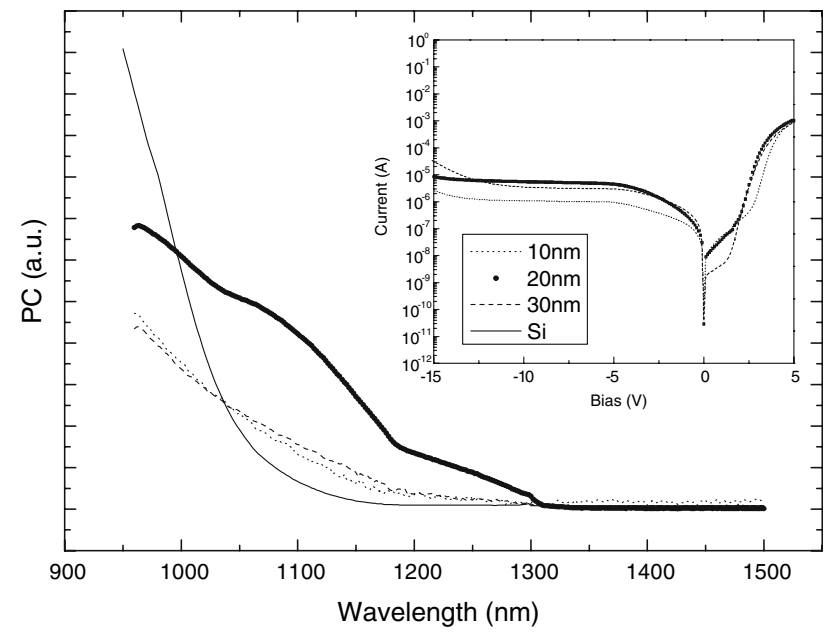

Fig. 3 Room temperature photocurrent of $\mathrm{Si} / \mathrm{SiGe}_{0.06}$ photodetectors under 0.5 reversed bias. Inset plot is $\mathrm{I}-\mathrm{V}$ characteristic of the devices

confinement effects. However, in these samples we see a further enhancement in the near IR absorption as a result of higher germanium concentrations that occur at the peaks of the corrugated structures. Interestingly, the highest PC is seen for the sample of intermediate germanium thickness $(20 \mathrm{~nm})$ rather than sample with the $30 \mathrm{~nm}$ thick germanium. We believe that relatively small changes in epitaxy conditions may be responsible for the seemingly unusual $20 \mathrm{~nm}$ Ge result. A simple empirical estimation of the $\mathrm{SiGe}$ composition at the crest of the corrugated structures is about $15 \%$ Ge in coherently strained regime, People et al. [1].

Indirect interband absorption in $\mathrm{Si}$ and $\mathrm{Ge}$ requires assistance of phonon emission and absorption processes. No-phonon transitions can be found in indirect material at only very low temperature. This optical process can be explained by using Macfarlane's model [17]. This formula can be fitted to experimental data by the plot of the square root of $I_{p h} \times h v$ versus udetected photon energy. The plot produces a straight line for $h v<E_{G}+E_{p h}$. The intercept of energy leads $E_{G}-E_{p h}$. Sbtracting the first square dependence from $I_{p h} \times h v$, finds $E_{G}+E_{p h}$. Therefore, $E_{G}$ and $E_{p h}$ can be determined by using these two intercepts [18]. However, this data fitting may give uncertainty due to square root plot. Table 1 shows energy gap and phonon energy from the devices. The energy gap seems to reduce with increased thickness and this can mainly be attributed to quantum confinement effects in $\mathrm{Si}_{1-x} \mathrm{Ge}_{x}$ nanostructures. The outcome phonon energy of 2675-1 and 2 stands between transverse optical phonon (TO) of Si-Ge $(50 \mathrm{meV})$ and $\mathrm{Ge}-\mathrm{Ge}(35 \mathrm{meV})[2]$, while the thickest device gives $65 \mathrm{meV}$, which is close to $\mathrm{TO}_{\mathrm{Si}-\mathrm{Si}}(58 \mathrm{meV})$. 


\section{Conclusion}

Near-infrared photodetectors were fabricated by using standard CMOS technology in combination with $\mathrm{Si}_{1-x} \mathrm{Ge}_{x}$ layers grown by LPCVD. The structure of $\mathrm{Si}_{1-x} \mathrm{Ge}_{x}$ layer exhibits corrugations that are attributed to $3 \mathrm{D}$ growth resulting from strain relief mechanisms. The crest of the layer is believed to contain a high Ge content, and this contributes to the relatively long wavelength photodetection. With optimization of germanium concentration and quantum well thickness, greater understanding and exploitation of self-organized quantum dot formation it should be possible to produce relatively inexpensive, CMOS compatible, communication wavelength $\mathrm{Si} / \mathrm{SiGe}$ photodetectors.

\section{References}

1. R. People, IEEE J. Quantum Electron. QE-22(9), 1696 (1986)

2. J. Weber, M.I. Alonso, Phys. Rev. B 40(8), 5683 (1989)
3. J.C. Sturm et al., Phys. Rev. Lett. 66(10), 1362-1365 (1991)

4. D.J. Robbins et al., J. Appl. Phys. 71(3), 1407 (1991)

5. J.C. Bean, Proc. IEEE 80(4), 571 (1992)

6. E. Corbin et al., Superlattice. Microst. 19(1), 25 (1996)

7. N.E.I. Etteh, P. Harrison, IEEE J. Quantum Electron. 37(5), 672 (2001)

8. R. Strong et al., J. Appl. Phys. 82(10), 5191 (1997)

9. G. Palfinger et al., Physica E, 2 (2002)

10. H. Lafontaine, J. Appl. Phys. 86(3), 1287 (1999)

11. S. Murtaza, IEEE J. Quantum Electron. 41(12), 2297 (1994)

12. S. Bozzo, J. Cryst. Growth, 216, 171 (2000)

13. D.J. Robbins et al., Appl. Phys. Lett. 66(12), 1512 (1995)

14. K. Nakajima et al., J. Cryst. Growth, 260, 372 (2004)

15. J.M. Bonar, Process Development and Characterization of Silicon and Silicon-Germanium Grown in a Novel Single-Wafer LPCVD System, in Electronics and Computer Sciences. (University of Souhampton, Southampton, 1995), pp. 114-117

16. M. Elkurdi et al., J. Appl. Phys. 92(4), 1858 (2002)

17. G.G. Macfarlane et al., Phys. Rev., 111(5), 1245 (1958)

18. A. Vonsovici et al., IEEE Trans Electron Dev. 45(2), 538 (1998) 